\title{
SIW pillbox antenna with integrated beam-switching feeds for mutual coupling reduction between close-spaced sources \\ E. Gandini ${ }^{1,2}$, M. Ettorre ${ }^{1}$, and R. Sauleau ${ }^{1}$ \\ ${ }^{1}$ IETR - Université de Rennes 1, Rennes, France. \\ ${ }^{2}$ LEMA - EPFL, Lausanne, Switzerland.
}

The beam overlapping level between adjacent beams is a very important factor in order to obtain an efficient coverage of the beam-scanning range using electrically switched multi-beam antennas. Typically, a $-3 \mathrm{~dB}$ beam-crossing level is desired. In a reflector system, this can be achieved by reducing the interspace distance between consecutive sources. By reducing the distance between adjacent feeds and eventually their size, the mutual coupling between them increases. Typically, a high level of mutual coupling can reduce the directivity of the sources, affecting the efficiency of the whole antenna system (Llombart et al., IEEE Trans. Antennas Propag., 56, 4, 1201-1206, 2008). Besides, in pillbox systems, the parabola taper and overall losses also increase.

The solution proposed in this work to achieve an efficient beam-crossing level and to keep low values of mutual coupling is a substrate integrated waveguide (SIW) leaky-wave feed. It consists of two partially reflecting grids made by vertical metallic pins connecting the upper and lower metallic plates of a parallel plate waveguide (PPW). The sources are the inner conductors of probe-like transitions. By properly choosing the geometrical dimensions of the partially reflecting grids, the leaky-wave modes propagating in the structure can be used to opportunely shape the field launched inside the PPW and considerably reduce the mutual coupling between the sources (Gandini et al., IEEE Antennas and Wireless Propag. Lett., 10, 647-650, 2011).

The pin-made feed is employed as a feed for a SIW pillbox system (Ettorre et al., IEEE Trans. Antennas Propag., 59, 4, 1093-1100, 2011). The pillbox, in this case, is excited with a one feed-per-beam beam-switching system. The radiating part is a slotted waveguide array. The quasi-optical transition is made of a SIW parabolic reflector and several coupling slots etched in the metal layer located between the two substrates hosting the input and radiating parts. The probe-like sources are placed in the focal plane of the parabola with an inter-distance $d_{s}=0.75 \lambda_{d}$, with $\lambda_{d}$ the wavelength in the dielectric at $f_{0}=24$ GHz.

The radiation patterns present a beam-crossing level of approximately $-3 \mathrm{~dB}$, as desired. The mutual coupling (scattering parameters) between adjacent sources is approximately $-20 \mathrm{~dB}$ at the central frequency. The mutual coupling remains under $-15 \mathrm{~dB}$ over a bandwidth of $\approx 10 \%$. This demonstrates the possibility to obtain a satisfactory beam-crossing level by keeping a very low value of mutual coupling between adjacent sources. 